\title{
Mitochondrial function is involved in regulation of cholesterol efflux to apolipoprotein (apo)A-I from murine RAW 264.7 macrophages
}

Anne Marie Allen and Annette Graham*

\begin{abstract}
Background: Mitochondrial DNA damage, increased production of reactive oxygen species and progressive respiratory chain dysfunction, together with increased deposition of cholesterol and cholesteryl esters, are hallmarks of atherosclerosis. This study investigated the role of mitochondrial function in regulation of macrophage cholesterol efflux to apolipoprotein A-l, by the addition of established pharmacological modulators of mitochondrial function.

Methods: Murine RAW 264.7 macrophages were treated with a range of concentrations of resveratrol, antimycin, dinitrophenol, nigericin and oligomycin, and changes in viability, cytotoxicity, membrane potential and ATP, compared with efflux of $\left[^{3} \mathrm{H}\right]$ cholesterol to apolipoprotein (apo) A-I. The effect of oligomycin treatment on expression of genes implicated in macrophage cholesterol homeostasis were determined by quantitative polymerase chain reaction, and immunoblotting, relative to the housekeeping enzyme, Gapdh, and combined with studies of this molecule on cholesterol esterification, de novo lipid biosynthesis, and induction of apoptosis. Significant differences were determined using analysis of variance, and Dunnett's or Bonferroni post $t$-tests, as appropriate.
\end{abstract}

Results: The positive control, resveratrol (24 h), significantly enhanced cholesterol efflux to apoA-I at concentrations $\geq 30 \mu \mathrm{M}$. By contrast, cholesterol efflux to apoA-I was significantly inhibited by nigericin $(45 \% ; p<0.01)$ and oligomycin (55\%; $p<0.01)$, under conditions $(10 \mu \mathrm{M}, 3 \mathrm{~h})$ which did not induce cellular toxicity or deplete total cellular ATP content. Levels of ATP binding cassette transporter A1 (ABCA1) protein were repressed by oligomycin under optimal efflux conditions, despite paradoxical increases in Abca1 mRNA. Oligomycin treatment did not affect cholesterol biosynthesis, but significantly inhibited cholesterol esterification following exposure to acetylated LDL, and induced apoptosis at $\geq 30 \mu \mathrm{M}$. Finally, oligomycin induced the expression of genes implicated in both cholesterol efflux (Abca1, Abcg4, Stard1) and cholesterol biosynthesis (Hmgr, Mvk, Scap, Srebf2), indicating profound dysregulation of cholesterol homeostasis.

Conclusions: Acute loss of mitochondrial function, and in particular $\Delta \psi_{m}$, reduces cholesterol efflux to apoA-I and dysregulates macrophage cholesterol homeostasis mechanisms. Bioavailable antioxidants, targeted to mitochondria and capable of sustaining effective mitochondrial function, may therefore prove effective in maintenance of arterial health.

Keywords: Cholesterol efflux, Cholesterol esterification, Liver X Receptor, ATP binding cassette transporter A1, Apolipoprotein A-I, High density lipoprotein

\footnotetext{
* Correspondence: Ann.Graham@gcu.ac.uk

Department of Life Sciences, School of Health and Life Sciences and the

Diabetes Research Group, Institute for Applied Health Research, Glasgow

Caledonian University, Cowcaddens Road, Glasgow G4 OBA, UK
} 


\section{Background}

Atherosclerosis is associated with increased production of reactive oxygen species (ROS) in mitochondria, accumulation of mitochondrial DNA damage, and progressive respiratory chain dysfunction [1-4]. In turn, mitochondrial dysfunction is induced by hypercholesterolemia, hyperglycemia, hypertriglyceridemia, and even the process of aging. Increased oxidative stress within the artery wall modifies low-density lipoprotein (LDL) to a form recognised by macrophage scavenger receptors, resulting in the unregulated uptake of excess cholesterol and cholesteryl esters within macrophage 'foam cells', a hallmark of early and developing atheroma [1-5].

Regression and stabilisation of atherosclerotic lesions requires the efficient removal of cholesterol from macrophage 'foam cells' to apolipoprotein (apo)A-I or apoE, allowing nascent high density lipoprotein (HDL) particles to enter and mature within the reverse cholesterol transport (RCT) pathway in the bloodstream, which delivers excess cholesterol to the liver for excretion as bile and bile acids [6]. Recently, we demonstrated that mitochondrial cholesterol transport is a key step controlling macrophage cholesterol efflux, the first step in the RCT pathway [7]. Notably, overexpression of steroidogenic acute regulatory protein (StAR; StarD1) in macrophages increases delivery of cholesterol to sterol 27-hydroxylase (CYP27A1), located on the inner mitochondrial membrane [7]. Sterol 27-hydroxylase generates oxysterol ligands for Liver X Receptors (LXR $\alpha / \beta)$; nuclear transcription factors which act as master regulators of genes encoding proteins involved in the cholesterol efflux pathway [8]. These include ATP binding cassette (ABC) transporters, such as ABCA1 and ABCG1/ABCG4, which work in concert to transfer cholesterol across the plasma membrane to apoA-I and HDL, respectively $[9,10]$.

High levels of sterol 27-hydroxylase are found to colocalise with macrophages in human atherosclerotic lesions [11], and 27-hydroxycholesterol is a major oxysterol in human atheroma [12]. The importance of CYP27A1 in macrophage cholesterol homeostasis is highlighted by the rare genetic disorder, cerebrotendinous xanthomasis, which is characterized by increased risk of premature atherosclerosis, despite normal concentrations of cholesterol in the bloodstream [13]. Importantly, the rate-limiting step controlling the activity of sterol 27hydroxylase is the supply of cholesterol to the enzyme, which can be mediated by the interaction of StAR with a protein complex located at contact sites between outer and inner mitochondrial membranes [7,14-16].

These findings, together with studies on mitochondrial cholesterol transport in steroidogenic tissues [16-19] led directly to our original hypothesis: that mitochondrial function be intrinsically linked to efficiency of macrophage cholesterol efflux, with consequences for other cholesterol homeostasis mechanisms. Certainly, transfer of cholesterol from the outer to the inner mitochondrial membrane requires energised, polarised and actively respiring mitochondria [16-20], while compounds such as resveratrol (3, 4, -5-trihydroxy-trans-stilbene), which protect mitochondrial function by inducing mitochondrial biogenesis [21] and reducing oxidative stress [22], are also reported to increase macrophage cholesterol efflux [22-24]. However, resveratrol is also reported to induce the expression of LXR $\alpha$ [23] and to impact positively on cholesterol flux in human cells via PPAR $\gamma$ and adenosine [24], suggesting pleiotropic modes of action for this molecule.

The aim of this study was to use established and specific modulators of mitochondrial function (antimycin, dinitrophenol, nigericin and oligomycin) to test whether a causal link exists between loss of mitochondrial function and impaired macrophage cholesterol efflux. Changes in cell viability and cytotoxicity, membrane potential and total ATP levels were compared with the ability of macrophages to efflux cholesterol to apoA-I; resveratrol was included as a putative positive control in these experiments, as this molecule is known to exert hormetic and cell-specific effects [25]. The results have implications for diseases associated with impaired mitochondrial function, including ageing, atherosclerosis, Alzheimer's disease and diabetes.

\section{Results}

The first part of this study set out to confirm the reported positive impact of resveratrol on cholesterol efflux, in particular under conditions of demonstrably unimpaired (or improved) mitochondrial function and sustained cellular viability [21-24]. The impact of resveratrol $(0-100 \mu \mathrm{M} ; 24 \mathrm{~h})$ on the conversion of MTT to formazan is shown in Figure 1A, with a significant decline in cell viability noted at $100 \mu \mathrm{M}$. Concentrations of $50 \mu \mathrm{M}(p<0.01)$ and $100 \mu \mathrm{M}(p<0.001)$ were associated with significantly increased release of cytosolic LDH into the medium (Figure 1B), and decreased Caspase 3/7 activity $(p<0.05)$ (Figure $1 C)$, suggesting cellular necrosis, rather than induction of apoptosis. Levels of ATP showed a similar pattern of decline, with a significant $58 \%$ reduction $(p<0.001)$ noted at $100 \mu \mathrm{M}$ resveratrol (Figure 1D). Interestingly, despite the proposed functions of resveratrol [21-24], this compound impacted negatively on membrane potential, with minor but reproducible losses noted at concentrations $\geq 30 \mu \mathrm{M}$ (Figure 1E), concentrations used previously to demonstrate cardioprotective properties for this compound [23]. As previously described [22], resveratrol significantly increased the efflux of $\left[{ }^{3} \mathrm{H}\right]$ cholesterol (Figure 1F) to apoA-I $\left(20 \mu \mathrm{g} \mathrm{ml}^{-1}\right)$, but only at the limit of this concentration range $(30 \mu \mathrm{M})$ and not at lower concentrations, when 

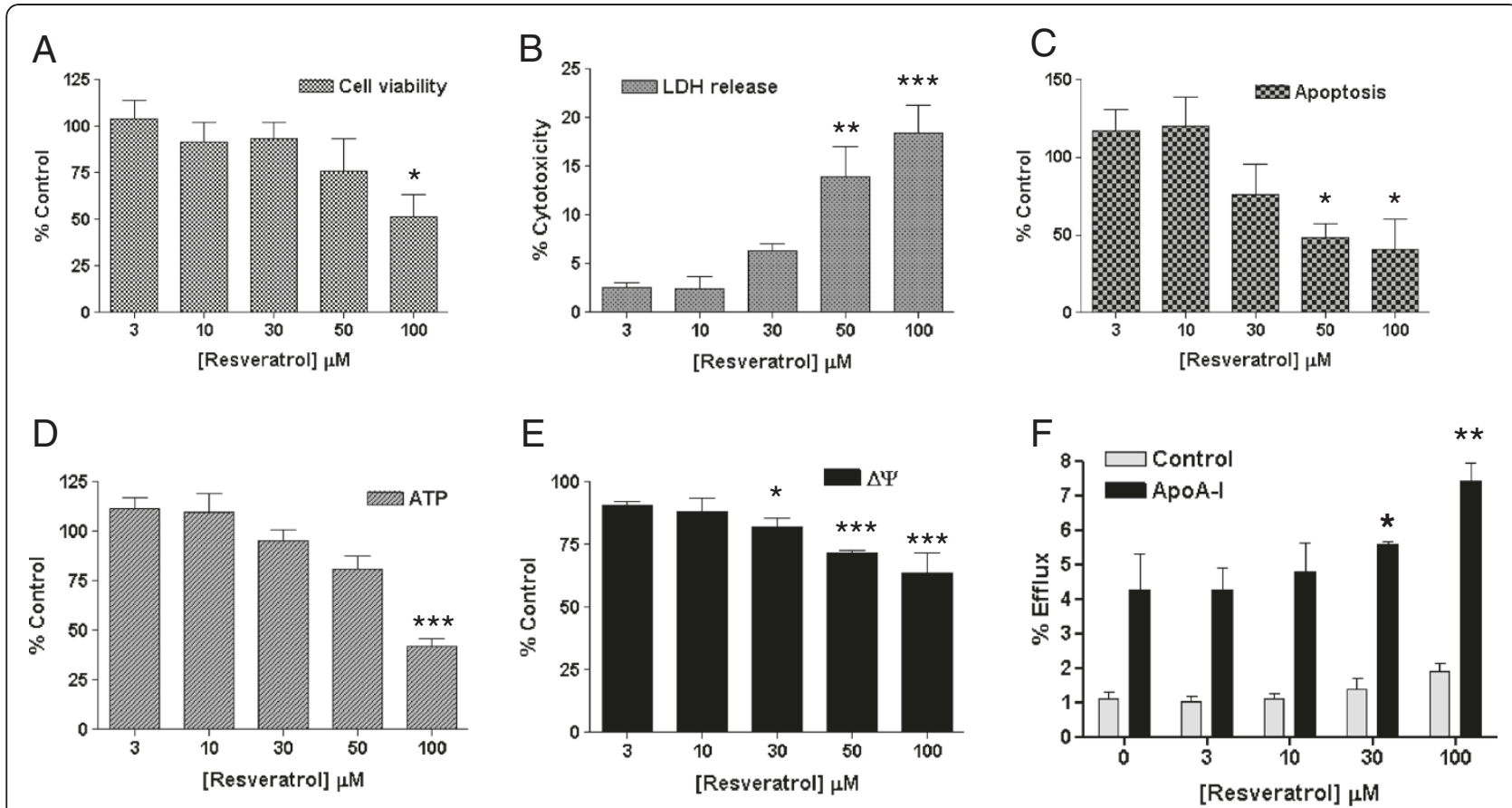

Figure 1 The effect of resveratrol, used at the concentrations indicated for 24 h on A) cell viability, B) cytotoxicity, C) apoptosis, D)

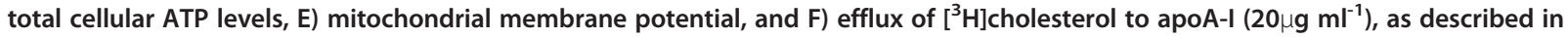
the Methods. Values are the mean \pm SEM of between three to four independent experiments; ${ }^{*} p<0.05 ;{ }^{* *} p<0.01$ and ${ }^{* * *} p<0.001$ compared with control macrophages.

tested under optimal efflux conditions (0.3 mM dibutyryl cAMP [20]). These findings therefore provide only limited evidence for a positive impact of this 'nutriceutical' on macrophage cholesterol efflux, suggesting that other properties of this compound may be more important in conferring cardioprotection [21-24].

The second part of this study therefore sought evidence that loss of mitochondrial function diminishes the atheroprotective cholesterol efflux pathway, again under conditions without overt loss of cell viability, release of cytosolic LDH or loss of total cellular ATP; reductions in mitochondrial membrane potential were, however, predicted for some compounds by previous studies [16-19,26], and formed part of the hypothesis under test. Mitochondrial inhibitors, nigericin and antimycin, decreased cellular viability (Figure 2A) and/or tended to increase release of cytosolic LDH into the medium (Figure $2 \mathrm{~B}$ ) at concentrations $\geq$ $30 \mu \mathrm{M}$ after $3 \mathrm{~h}$, while dinitrophenol or oligomycin had no significant impact on either parameter during this period. By contrast, more prolonged incubation $(24 \mathrm{~h})$ with these inhibitors caused substantive loss of cell viability, with estimated $\mathrm{LD}_{50}$ values of $1 \mu \mathrm{M}$ (antimycin), $75 \mu \mathrm{M}$ (dinitrophenol), $1 \mu \mathrm{M}$ (nigericin) and $8 \mu \mathrm{M}$ (oligomycin), as judged by MTT conversion to formazan (data not shown). During a $3 \mathrm{~h}$ incubation, intracellular levels of ATP were depleted by oligomycin $(30 \mu \mathrm{M})$ and nigericin $(\geq 10 \mu \mathrm{M})$ by $24 \%$ $(p<0.01)$ and $28 \%(p<0.001)$, respectively, but not by the other drugs tested (Figure $2 \mathrm{C}$ ), while mitochondrial membrane potential declined significantly by $39 \%(p<0.05)$ only in the presence of oligomycin $(\geq 10 \mu \mathrm{M})$.

When tested at concentrations which did not affect cellular viability $(10 \mu \mathrm{M} ; 3 \mathrm{~h})$, only nigericin and oligomycin inhibited efflux of $\left[{ }^{3} \mathrm{H}\right]$ cholesterol to apoA-I (Figure 3A) by $45 \%(p<0.01)$ and $55 \%(p<0.01)$, respectively, following preincubation with dibutyryl cAMP $(0.3 \mathrm{mM})$. By contrast, neither antimycin nor dinitrophenol significantly changed cholesterol efflux to apoA-I. While macrophage levels of ABCA1 protein were modestly increased following oligomycin treatment, levels of this protein were repressed by oligomycin $(34 \pm 17 \% ; n=3 ; p<0.05)$ in macrophages incubated under optimal efflux conditions ( $0.3 \mathrm{mM}$ dibutyryl cAMP and $20 \mu \mathrm{g} \mathrm{ml}^{-1}$ apoA-I), and by comparison with GADPH (Figure 3B).

Oligomycin treatment induced gene expression of Abcal and Abcg4 mRNA, together with genes encoding other enzymes and transcription factors involved in cholesterol metabolism, including Hmgcr, Mvk, Scap1, Srebf1, Srebf2 and Stard1 (Figure 4A). Further, when macrophages were incubated in the presence of acetylated LDL $\left(50 \mu \mathrm{g} \mathrm{ml}^{-1}\right)$ to induce 'foam cell' formation, oligomycin $(10 \mu \mathrm{M} ; 3 \mathrm{~h})$ inhibited acyl CoA: cholesterol acyltransferase (ACAT 1$)$ activity by $35 \%(p<0.01)$, as judged by incorporation of $\left[{ }^{3} \mathrm{H}\right]$ oleate $(10 \mu \mathrm{M})$ into the cholesteryl ester pool (Figure 4B). Equally, under basal 

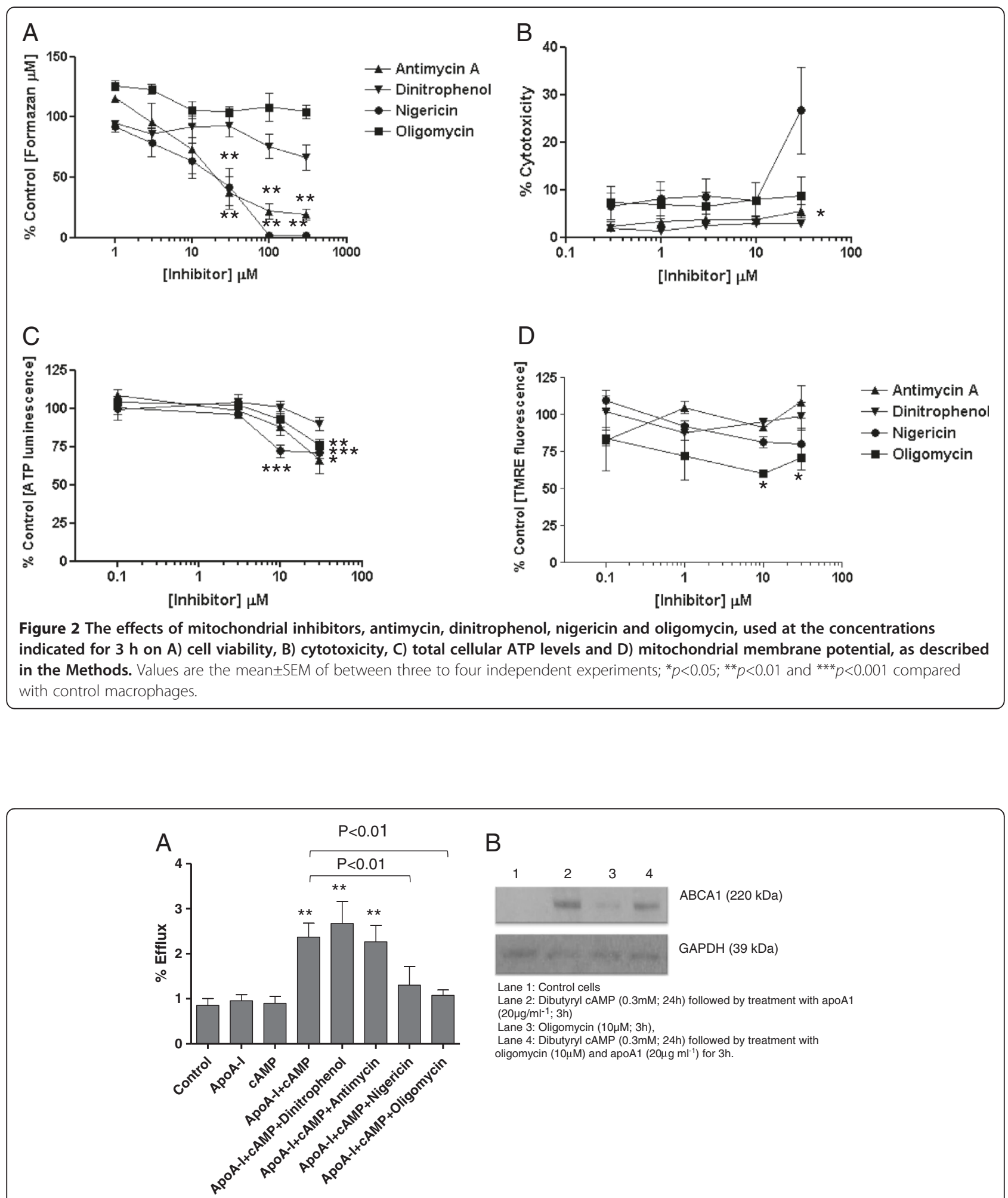

B

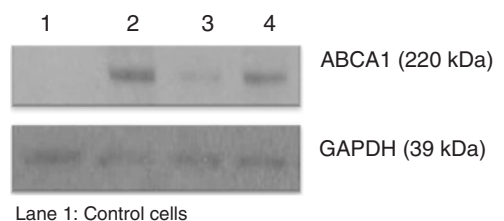

Lane 1: Control cells

Lane 2: Dibutyryl cAMP $(0.3 \mathrm{mM} ; 24 \mathrm{~h})$ followed by treatment with apoA1

$\left(20 \mu \mathrm{g} / \mathrm{ml}^{-1} ; 3 \mathrm{~h}\right)$

Lane 3: Oligomycin $(10 \mu \mathrm{M} ; 3 \mathrm{~h})$

Lane 4: Dibutyryl cAMP $(0.3 \mathrm{mM} ; 24 \mathrm{~h})$ followed by treatment with

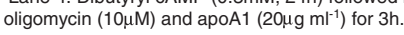

Figure 3 The effects of mitochondrial inhibitors, antimycin, dinitrophenol, nigericin and oligomycin, each used at 10 $\mu \mathrm{M}$ for $3 \mathrm{~h}$, on efflux of $\left[^{3} \mathrm{H}\right]$ cholesterol to apoA-I $\left(20 \mathbf{~ g ~ m ~ m}^{-1}\right)$ is shown in Figure 3A. Where indicated, macrophages were preincubated for $24 \mathrm{~h}$ in the presence of dibutyryl CAMP $(0.3 \mathrm{mM})$ to induce expression of ABCA1 and cholesterol efflux [25]. Values are the mean \pm SEM of between four to six independent experiments; ${ }^{* *} p<0.01$ compared with control macrophages; other significant changes are indicated on the figure. Expression of ABCA1 protein under the conditions noted is shown in Figure 3B; values are shown relative to the housekeeping gene, GAPDH, and are representative of three independent experiments. 

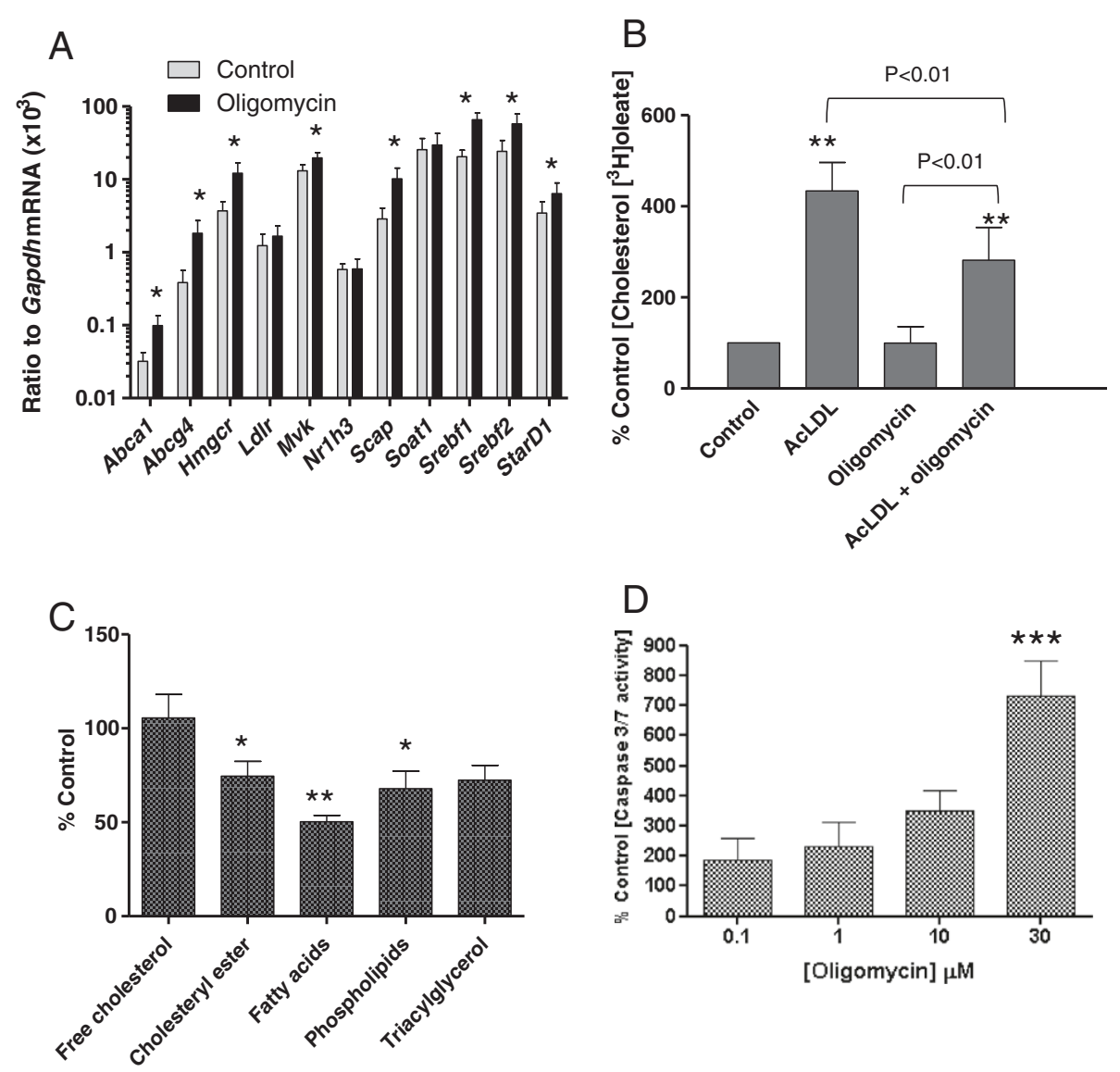

Figure 4 The effect of oligomycin ( $10 \mu \mathrm{M} ; 3 \mathrm{~h})$ on A) fold-change of genes involved in cholesterol metabolism and lipoprotein signalling in RAW 264.7 macrophages, compared with control cells, as judged by Q-PCR analysis of genes implicated in the cholesterol efflux, biosynthesis and esterification pathways; B) incorporation of $\left[{ }^{3} \mathrm{H}\right]$ oleate $(10 \mu \mathrm{M})$ into the cholesteryl ester pool, measured in the presence of absence of acetylated LDL $\left.\left(50 \mu \mathrm{g} \mathrm{ml}^{-1} ; 3 \mathrm{~h}\right) ; \mathrm{C}\right)$ incorporation of $\left[{ }^{14} \mathrm{C}\right]$ acetate into free cholesterol, cholesteryl ester, fatty acids, phospholipids and triacylglycerol $(\mathbf{3 ~ h}$ ) and D) induction of apoptosis in RAW $\mathbf{2 6 4 . 7}$ murine macrophages. Values are the mean \pm SEM of between three to six independent experiments. ${ }^{*} p<0.05,{ }^{* *} p<0.01$ and ${ }^{* *} p<0.001$ compared with control macrophages.

conditions, oligomycin $(10 \mu \mathrm{M} ; 3 \mathrm{~h})$ significantly decreased incorporation of $\left[{ }^{14} \mathrm{C}\right]$ acetate into cholesteryl ester, fatty acid and phospholipid pools, while biosynthesis of free $\left[{ }^{14} \mathrm{C}\right]$ cholesterol remained unaffected (Figure $4 \mathrm{C}$ ). Finally, increasing concentrations of oligomycin were associated with a trend towards increasing macrophage apoptosis (Figure 4D), which proved significant at $30 \mu \mathrm{M}$.

\section{Discussion}

Loss of mitochondrial function, a key element of atherogenesis, is caused by an increase in reactive oxygen species, resulting in accumulation of mitochondrial DNA damage, and respiratory chain deficiencies, apoptosis and cell death, favouring plaque formation and instability [1-5]. This study argues that dysregulation of macrophage cholesterol homeostasis should also join this list, apparently driven by the specific loss of macrophage cholesterol efflux to apoA-I.
Notably, resveratrol, a natural phytoalexin antioxidant found in grapes and red wine, known to induce mitochondrial biogenesis and protect against atherosclerosis in animal models [23,25], increased cholesterol efflux to apoA-I, at concentrations around $20-30 \mu \mathrm{M}$, as previously described $[22,24]$. However, it is evident that concentrations of resveratrol $(30-100 \mu \mathrm{M})$ which increase cholesterol efflux (Figure 1), and indeed those reported to activate LXR $\alpha$ [23], are associated with loss of mitochondrial membrane potential and cell viability and induction of cytotoxicity in RAW 264.7 macrophages. Moreover, since resveratrol is characterised by high absorption but very low bioavailability, coupled with extensive and rapid metabolism to conjugates of this compound [27], it seems unlikely that monocyte-macrophages would be exposed to the concentrations of resveratrol needed to stimulate the cholesterol efflux process. For example, absorption (70\%) of a dietary relevant $25 \mathrm{mg}$ oral dose generated peak plasma levels of around $2 \mu \mathrm{M}$ resveratrol metabolites, with 
only trace amounts of unchanged resveratrol detected in the plasma $\left(<5 \mathrm{ng} \mathrm{ml}^{-1}\right)$ [27]. However, it may be possible that the more potent antioxidant function of resveratrol $(\leq 5 \mu \mathrm{M})$ [22], or the ability of this compound to amplify SIRT-1 dependent biogenesis of mitochondria $(\leq 10 \mu \mathrm{M})$ [21], might help to sustain mitochondrial function in vivo, particularly if local depots of resveratrol accumulate within arterial cells. If so, macrophages may have to be oxidatively challenged, or subject to chronic incubation with resveratrol, for the protective impact of this compound on the cholesterol efflux pathway to become more (patho)physiologically relevant.

By contrast, inhibitors of differing aspects of mitochondrial function $\left(\Delta \mathrm{pH}, \Delta \psi_{\mathrm{m}}\right.$ complex III or complex V) exerted convincing, if selective, effects on the cholesterol efflux pathway over the acute time period tested here. Notably, the uncoupling agent, dinitrophenol, and complex III inhibitor, antimycin, had negligible impact on efflux to apoA-I, while nigericin and oligomycin both reduced efflux to apoA-I. While nigericin $(10 \mu \mathrm{M})$ decreased total cellular ATP levels, which could impact on the activity of $\mathrm{ABC}$ transporters, $\mathrm{ABCA} 1$ and $\mathrm{ABCG} 1 / \mathrm{G} 4$, oligomycin did not alter cell viability or total ATP levels, but did significantly reduce $\Delta \psi_{\mathrm{m}}$, unlike the other inhibitors tested. In turn, loss of $\Delta \psi_{\mathrm{m}}$ due to oligomycin was associated with inhibition of cholesterol esterification and a trend towards induction of apoptosis, together with increased expression of an array of genes involved in cholesterol homestasis, arguing a profound dysregulation of cholesterol homeostasis as sequelae of acute loss of mitochondrial function. Importantly, the coordinated response which should link impaired cholesterol efflux with SREBP-2 repression of cholesterol biosynthesis and LDL uptake, and with increased cholesterol esterification, seems to be lost.

While this is the first study to focus on the importance of mitochondria in regulation of macrophage cholesterol efflux, related studies have been performed in steroidogenic cells $[16-19 ; 26]$. As previously introduced, the rate-limiting step in the generation of steroid hormones, is the transfer of cholesterol into mitochondria, to the CYP11A1 protein which resides on the inner mitochondrial membrane. Dissipation of $\Delta \psi_{\mathrm{m}}$ with CCCP, inhibition of electron transport using antimycin $\mathrm{A}$, disruption of $\mathrm{pH}$ using nigericin, and inhibition of $\mathrm{F}_{0} / \mathrm{F}_{1}$ ATP synthase using oligomycin, all inhibited progesterone synthesis in Leydig cells, indicating that altered mitochondrial function regulates steroid biosynthesis [16-19; 26]. However, in RAW 264.7 macrophages, only nigericin and oligomycin regulated macrophage cholesterol efflux to apoA-I, and at concentrations 10-fold higher than those required to inhibit steroidogenesis $[16-19,26]$. It is noteworthy that cellular ATP content was more sensitive to depletion by mitochondrial disruption in Leydig cells than in RAW 264.7 macrophages, as only oligomycin $(\geq 30 \mu \mathrm{M})$ and nigericin $(\geq 10 \mu \mathrm{M})$ reduced cellular ATP levels over the same time scale in the latter; equally, $\Delta \psi_{\mathrm{m}}$ was markedly reduced by antimycin $(1 \mu \mathrm{M})$ and oligomycin $(1 \mu \mathrm{M})$ in Leydig cells, while only oligomycin $(\geq 10 \mu \mathrm{M})$ affected this parameter in the current study. This may explain the apparent selectivity for nigericin and oligomycin in repression of cholesterol efflux to apoA-I; certainly, loss of either $\Delta \psi_{\mathrm{m}}$ or cellular ATP seems sufficient to negatively affect macrophage cholesterol efflux when cell viability is sustained (Figures 2 and 3).

Oligomycin treatment, by limiting cholesterol efflux (Figure 2A) and reducing cholesterol esterification (Figure 4B), without impacting on cholesterol biosynthesis (Figure 4C), should therefore lead to accumulation of sterol at the endoplasmic reticulum (ER). This should trigger a protective cholesterol homeostasis response, sequestering SREBPs at the ER, and providing oxysterol ligands for Liver X Receptors (LXRs) complexed with retinoid X receptors (RXR) at the LXR response element within genes involved in the cholesterol efflux pathway [8]. Sterol-dependent sequestration of SREBPs at the ER prevents proteolytic processing of this transcription factor to the nuclear SREBP fragment capable of inducing the transactivation of genes involved in cholesterol biosynthesis and uptake. However, instead of these correctly orchestrated events, treatment with oligomycin resulted in upregulation of genes involved in both cholesterol biosynthesis (Hmgr, Mvk, Scap, Srebf1, Srebf2) and efflux (Abca1, Abcg4, StarD1) pathways, arguing a profound dysregulation of this response. The mechanism involved was not investigated here, but it is possible to speculate that loss of mitochondrial production of oxysterol LXR ligands [7] may release SREBP2 from the ER, explaining the increased expression of genes involved in increasing cholesterol biosynthesis. This could then result in increased production of 24(S),25-epoxycholesterol via the cholesterol biosynthetic pathway [28], facilitating LXR activation and induction of genes involved in the efflux process.

One further outcome predicted by the macrophage lipid phenotype observed following oligomycin treatment is toxic overaccumulation of cholesterol at the ER, which can trigger oxidative stress and proteasomal degradation of existing ABCA1 protein [29] and ultimately trigger apoptosis, observed at higher concentrations of oligomycin (Figure 4D). While it is clear that oligomycin induction of Abcal mRNA may explain the modest increase in $A B C A 1$ protein under basal conditions, a clear reduction in ABCA1 is noted when macrophages are treated with this inhibitor under optimal efflux conditions (Figure 3B), which agrees well with the observed loss of $\left[{ }^{3} \mathrm{H}\right]$ cholesterol efflux to apoA-I under this 
condition (Figure 3A). The dissociation between expression of ABCA1 mRNA and protein in oligomycintreated macrophages under this condition reflects that observed in carotid atherosclerotic lesions, where elevated Abca1 mRNA is associated with reduced expression of ABCA1 protein [30].

\section{Conclusions}

Loss of mitochondrial function impacts negatively on macrophage cholesterol homeostasis, reducing efflux to apoA-I and impairing cholesterol esterification, events which may trigger accumulation of toxic free cholesterol and induction of apoptosis within arterial macrophages, contributing to lesion development. Small drug molecules, specifically targeted to accumulate within the mitochondria [31] and capable of sustaining $\Delta \psi_{\mathrm{m}}$ might, therefore, not only reduce arterial oxidative stress, but also improve cholesterol homeostasis and efflux from macrophage 'foam' cells, regressing and stabilising atherosclerotic plaque.

\section{Methods \\ Materials}

Murine RAW 264.7 macrophages were purchased from the European Collection of Cell Cultures (ECACC; Health Protection Agency Culture Collection, Porton Down, UK). Tissue culture reagents were purchased from Lonza (Wokingham, UK); other sources include FuGene6 transfection reagent (Roche), NuPAGE gels and buffers (InVitrogen), antibodies (AbCAM, Cambridge, UK), primers and probes (Eurogentec, Belgium). Athens Research (USA) provided apoA-I and low density lipoprotein (LDL); LDL was acetylated according to Brown et al. [32]. Radiolabels $\left(\left[{ }^{3} \mathrm{H}\right]\right.$ cholesterol and $\left[{ }^{14} \mathrm{C}\right]$ acetic acid) were provided by ICN Biologicals; all other chemicals were provided by Sigma-Aldrich (Poole, Dorset, UK).

\section{Cell Culture}

Murine RAW 264.7 macrophages were sustained using a split ratio of 1:4, in Dulbecco's Modified Eagles Medium (DMEM) supplemented with foetal bovine serum (FBS, $10 \%, \mathrm{v} / \mathrm{v}), \mathrm{L}$-glutamine $(200 \mathrm{mM})$ and penicillin/streptomycin $\left(50 \mu \mathrm{g} \mathrm{ml}^{-1} ; 50 \mathrm{U} \mathrm{ml}^{-1}\right.$, respectively). For experiments, cells were plated onto 12 -well tissue culture dishes at an initial density of $0.5 \times 10^{6}$ cells well $^{-1}$, under serum-free conditions, except where stated. Compounds with an established role in modulation of mitochondrial function (resveratrol, antimycin, dinitrophenol, nigericin and oligomycin) were added using DMSO $(<0.01 \%)$ as vehicle, at the concentrations and time periods indicated in the Legends to Figures.

\section{Macrophage lipid analysis and cholesterol efflux}

Incorporation of $\left[{ }^{14} \mathrm{C}\right]$ acetic acid $\left(1 \mu \mathrm{Ci} \mathrm{ml}{ }^{-1}\right)$ into fatty acid, phospholipid, cholesterol, cholesteryl ester and triacylglycerol pools were measured after incubation for $3 \mathrm{~h}$ in the presence of oligomycin $(10 \mu \mathrm{M})$, as previously described $[7,33]$. Esterification of cholesterol, in the presence of acetylated LDL $\left(50 \mu \mathrm{g} \mathrm{ml}^{-1}\right)$ was monitored by flux of $\left[{ }^{3} \mathrm{H}\right]$ oleic acid $\left(10 \mu \mathrm{M} ; 1 \mu \mathrm{Ci} \mathrm{ml}^{-1}\right)$ into cholesteryl $\left[{ }^{3} \mathrm{H}\right]$ oleate, as previously [33]. Macrophage lipids were extracted using hexane:isopropanol $(3: 2, \mathrm{v} / \mathrm{v})$ and dried under nitrogen, before separation by t.l.c. using chloroform, methanol and water (60:30:5, by vol.) as the first mobile phase, and hexane, diethyl ether and acetic acid (80:20:1.5, by vol.) in the second phase of development; lipids were identified by co-migration with authentic standards [7,33].

Macrophage efflux of $\left[{ }^{3} \mathrm{H}\right]$ cholesterol to apoA-I and HDL were determined as previously described [7,33]. Efflux from radiolabelled macrophages, was initiated by addition of serum-free DMEM containing human apoAI $\left(20 \mu \mathrm{g} \mathrm{ml}^{-1}\right)$ or HDL $\left(20 \mu \mathrm{g} \mathrm{ml}^{-1}\right)$ for $3 \mathrm{~h}$; preincubation (24 h) with dibutyryl cAMP $(0.3 \mathrm{mM})$ is required in this cell line to induce ABCA1, as previously described [20]. Results are expressed as \% cholesterol efflux, calculated as $\left[\mathrm{dpm}_{(\text {media })} / \mathrm{dpm}_{(\text {media+cells })}\right] \times 100 \%$.

\section{Analysis of mRNA and protein expression}

Total RNA was isolated (TriZol) from macrophages, and reverse transcribed in cDNA (BioLine) prior to measurement of cellular levels of Abca1, Ldlr, Mvk, Nr1h3, Scap, Soat1, Srebf1 and Srebf2 by quantitative polymerase chain reaction (Q-PCR), relative to Gapdh, using primers and fluorescent (FAM/TAMRA) probe sequences, and protocols cited in [7]. Levels of Abcg4 were determined using forward (5'-GCGCCTGGCCATTGC-3'), reverse (5'-GACCGCTGGTAGGCTCATCA-3') primer and probe (5'-CTGGTCAACAACCCGCCTGTCATGT3 ') sequences $(100 \mathrm{nM})$.

Macrophage cell lysates were prepared in RIPA buffer (25 mM Tris $\mathrm{HCl}$ pH7.6, $150 \mathrm{mM} \mathrm{NaCl}, 1 \%$ (v/v) NP-40, $1 \%(\mathrm{w} / \mathrm{v})$ sodium deoxycholate, $0.1 \%(\mathrm{w} / \mathrm{v})$ sodium dodecyl sulphate) supplemented with Complete Com $^{\mathrm{TM}}$ protease inhibitor cocktail (Roche), and proteins (50 $\mu \mathrm{g}$ lane $^{-1}$ ) separated by SDS PAGE (NuPAGE; $10 \%$ gels), transferred to a PDVF membrane and probed using anti-ABCA1 (1:1000) and anti-GAPDH (1:2000) rabbit polyclonal antibodies, and detection achieved using secondary antibodies coupled to the ECL detection system $[7,33]$.

\section{Assessment of macrophage viability, apoptosis and mitochondrial function}

Cell viability was assessed by conversion of methyl thiazolyl blue tetrazolium bromide to formazan, via succinate: 
ubiquinol oxidoreductase (Complex II), as previously [33], and by release of lactate dehydrogenase into the medium (LDH Cytotoxicity Assay Kit, Cambridge Biosciences). Membrane potential was assessed by tetramethylrhodamine fluorescence [17], total cellular ATP using the luciferase Celltiter Glo-Bioluminescent reagent supplied by Promega, and apoptosis by monitoring Caspase 3/7 activity (Promega).

All values indicate mean \pm S.E.M., with numbers of independent experiments denoted by $n$. Significant $(p<0.05)$ differences were determined using analysis of variance and Dunnett's, or Bonferroni post $t$-tests, as appropriate.

\section{Competing interests}

The authors declare that they have no competing interests.

\section{Author's contributions}

AMA carried out the experimental work on this study, which was jointly designed by AMA and AG. The manuscript was prepared by AG. Both authors have read and approved the final manuscript.

Received: 6 June 2012 Accepted: 4 December 2012 Published: 10 December 2012

\section{References}

1. Chang J-C, Kou S-J, Lin W-T, Liu C-S: Regulatory role of mitochondria in oxidative stress and atherosclerosis. World J. Cardiol 2010, 2:150-159.

2. Puddu P, Puddu GM, Cravero E, De Pascalis S, Muscari A: The emerging role of cardiovascular risk factor-induced mitochondrial dysfunction in atherogenesis. J. Biomed Sci 2009, 16:112. http://www.jbiomedsci.com/ content/16/1/112.

3. Madamanchi NR, Runge MS: Mitochondrial dysfunction in atherosclerosis. Circ Res. 2007, 100:460-473.

4. Mercer JR, Cheng K-K, Figg N, Gorenne I, Mahmoudi M, Griffin J, Vidal-Puig A, Logan A, Murphy MP, Bennett M: DNA damage links mitochondrial dysfunction to atherosclerosis and the metabolic syndrome. Circ Res. 2010, 107:1021-1031.

5. Gerrity RG: The role of the monocyte in atherogenesis. Am. J. Path. 1981, 103:181-190.

6. Kannel WM: High density lipoproteins: epidemiologic profile and risks of coronary artery disease. Am. J Cardiol. 1983, 52:9B-12B.

7. Taylor JMW, Borthwick F, Bartholomew C, Graham A: Overexpression of steroidogenic acute regulatory protein increases macrophage cholesterol efflux to apolipoprotein A-I. Cardiovasc. Res. 2010, 86:526-534.

8. Venkateswaran A, Lafitte BA, Joseph MB, Mak PA, Wilpitz DC, Edwards PA, Tontonez P: Control of cellular cholesterol efflux by the nuclear oxysterol receptor, LXR alpha. Proc Natl Acad Sci USA 2000, 97:12097-12102.

9. Oram JF, Heinecke JW: ATP-binding cassette transporter A1: a cell cholesterol exporter that protects against cardiovascular disease. Physiol. Rev. 2005, 85:1343-1372.

10. Wang N, Lan D, Chen W, Matsuura F, Tall AR: ATP-binding cassette transporters G1 and G4 mediate cellular cholesterol efflux to highdensity lipoproteins. Proc Natl Acad. Sci USA 2004, 101:9774-9779.

11. Crisby M, Nilsson J, Kostulas V, Bjorkhem I, Diczfalusy U: Localisation of sterol 27-hydroxylase immunoreactivity in human atherosclerotic plaques Biochim. Biophys Acta 1997, 1344:278-285.

12. Garcia-Cruset S, Carpenter KLH, Guardiola F, Stein BK, Mitchinson MJ: Oxysterol profiles of normal human arteries, fatty streaks and advance lesions. Free Rad. Res. 2001, 35:31-41.

13. Gallus GN, Dotti MT, Federico A: Clinical and molecular diagnosis of cerebrotendinous xanthomatosis with a review of the mutations in the CYP27A1 gene. Neurological Sciences 2006, 27:143-149.

14. Stocco DM: Tracking the role of a star in the sky of a new millennium. Mol Endocrinol. 2001, 15:1245-54.

15. Miller WL: Mechanism of StAR's regulation of mitochondrial import. Mol Cell Endocrinol. 2007, 265-266:46-50.
16. Rone MB, Fan J, Papadopoulos V: Cholesterol transport in steroid biosynthesis: role of protein-protein interactions and implications in disease states. Biochim. Biophys. Acta 2009, 1791:646-658.

17. Allen JA, Shankara T, Janus P, Buck S, Diemer T, Hales KH, Hales DB: Energized, polarized and actively respiring mitochondria are required for acute Leydig cell steroidogenesis. Endocrinol. 2006, 147:3924-3935.

18. Bose HS, Whittal RM, Baldwin MA, Miller WL: The active form of steroidogenic acute regulatory protein, StAR, appears to be a molten globule. Proc Natl. Acad. Sci USA 1999, 96:7250-55.

19. Yaworsky DC, Yang BB, Bose HS, Best KB, Jensen LB, Bell JD, Baldwin MA, Miller WL: pH-dependent interactions of the carboxy terminal helix of steroidogenic acute regulatory protein (StAR) with synthetic membranes. J Biol. Chem. 2004, 280:2045-54.

20. Oram JF, Lawn RM, Garvin MR, Wade DP: ABCA1 is the CAMP-inducible apolipoprotein receptor that mediates cholesterol secretion from macrophages. J. Biol. Chem. 2000, 275:34508-34511.

21. Csiszar A, Labinsky N, Pinto JT, Ballabh P, Zhang H, Losonczy G, Pearson K, de Cabo R, Pacher P, Zhang C, Ungvari Z: Resveratrol induces mitochondrial biogenesis in endothelial cells. Am J Physiol Heart Circ Physiol 2009, 297:H13-H2O

22. Berrougui H, Grenier G, Loued S, Drouin G, Khalil A: A new insight into resveratrol as an atheroprotective compound: inhibition of lipid peroxidation and enhancement of cholesterol efflux. Atherosclerosis 2009, 207:420-427.

23. Sevov M, Elfineh L, Cavelier LB: Resveratrol regulates the expression of LXR-alpha in human macrophages. Biochem. Biophys. Res. Commun. 2006, 348:1047-54.

24. Voloshyna I, Hai O, Littlefield MJ, Carsons S, Reiss AB: Resveratrol mediates anti-atherogenic effects on cholesterol flux in human macrophages and endothelial via PPARy and adenosine. Eur J Pharmacol. 2012, [Epub ahead of print].

25. Calabrese EJ, Mattson MP, Calabrese V: Dose response biology: The case of resveratrol. Hum Exp Toxicol 2010, 29:1034.

26. Hales DB, Allen JA, Shakara T, Janus P, Buck S, Diemer T, Hales KH: Mitochondrial function in Leydig cell steroidogenesis. Ann. N. Y. Acad Sci 2005, 1061:120-134.

27. Nakata $\mathrm{R}$, Takahashi $\mathrm{S}$, Inoue $\mathrm{H}$ : Recent advances in the study on resveratrol. Biol Pharm. Bull 2012, 35:273-279.

28. Wong J, Quinn CM, Brown AJ: SREBP-2 positively regulates transcription of the cholesterol efflux gene, $A B C A 1$ by generating oxysterol ligands for LXR. Biochem. J 2006, 400:485-491.

29. Feng B, Tabas I: ABCA1-mediated cholesterol efflux is defective in free cholesterol-loaded macrophages. J. Biol. Chem. 2002, 277:43271-80.

30. Albrecht C, Soumian S, Amey JS, Sardini A, Higgins CF, Davies AH, Gibbs RG: ABCA1 expression in carotid atherosclerotic plaques. Stroke 2004, 35:2801-2806

31. Armstrong JS: Mitochondrial medicine: Pharmacological targeting of mitochondria in disease. Br J Pharmacol, :1154-1165.

32. Brown MS, Basu SK, Falck JR, Ho YK, Goldstein JL: The scavenger cell pathway for lipoprotein degradation: specificity of the binding site that mediates the uptake of negatively-charged LDL by macrophages. $J$. Supramol Struct. 1980, 13:67-81.

33. Borthwick F, Allen AM, Taylor JM, Graham A: Overexpression of STARD3 in human monocyte-macrophages induces an anti-atherogenic lipid phenotype. Clinical Science 2010, 119:265-272

doi:10.1186/1476-511X-11-169

Cite this article as: Allen and Graham: Mitochondrial function is involved in regulation of cholesterol efflux to apolipoprotein (apo)A-I from murine RAW 264.7 macrophages. Lipids in Health and Disease 2012 11:169. 